\title{
Selecting Resistance to Bipolaris sorokiniana and Fusarium graminearum in Intermediate Wheatgrass
}

J. M. Krupinsky, Plant Pathologist, and J. D. Berdahl, Research Geneticist, United States Department of Agriculture-Agricultural Research Service, Northern Great Plains Research Laboratory, P.O. Box 459, Mandan, ND 58554-0459

\begin{abstract}
Krupinsky, J. M., and Berdahl, J. D. 2000. Selecting resistance to Bipolaris sorokiniana and Fusarium graminearum in intermediate wheatgrass. Plant Dis. 84:1299-1302.

Forage yield and persistence of intermediate wheatgrass and pubescent intermediate wheatgrass can be adversely affected by foot rot caused by Fusarium graminearum, Bipolaris sorokiniana, or both. Putative B. sorokiniana- and F. graminearum-resistant plants were selected from both Reliant and Manska cultivars of intermediate wheatgrass to produce four populations. When plants from half-sib families that were selected for resistance were intercrossed and progeny subjected to subsequent selection cycles for B. sorokiniana and F. graminearum resistance, progressively higher percentages of putative resistant seedlings were found for three of the four selected populations compared to the original base populations. In cycle 3 of three populations, 7 to $91 \%$ of half-sib families with statistically higher seedling survival rates than the base populations were considered more resistant than the base populations. Thus, progressive improvement in seedling survival was realized for three populations selected for resistance using three cycles of recurrent phenotypic selection among and within half-sib families.
\end{abstract}

Intermediate wheatgrass (Thinopyrum intermedium subsp. intermedium (Host) Barkw. \& Dewey) and pubescent intermediate wheatgrass (Thinopyrum intermedium subsp. barbulatum (Schur) Barkw. \& Dewey) are introduced cool-season grasses that are widely grown in the northern Great Plains of the United States and the grassland regions of western Canada. Dry matter yields are excellent and nutritive quality is good prior to heading (9). Crown rot and foot rot diseases have been reported on intermediate wheatgrass and other forage grasses $(3,6,10,11)$. Forage yield and persistence of intermediate wheatgrass can be adversely affected by foot rot diseases, particularly those caused by Bipolaris sorokiniana and by Fusarium graminearum Schwabe, teleomorph: Gibberella zeae (Schwein.) Petch (unpublished). In initial

Corresponding author: J. M. Krupinsky

E-mail: krupinsj@mandan.ars.usda.gov

Mention of a trademark, proprietary product, or company by USDA personnel is intended for explicit description only and does not constitute a guarantee or warranty of the product by the USDA and does not imply its approval to the exclusion of other products that may also be suitable. USDAARS, Northern Plains Area, is an equal opportunity/affirmative action employer and all agency services are available without discrimination.

Accepted for publication 18 August 2000.

Publication no. D-2000-0928-01R

This article is in the public domain and not copyrightable. It may be freely reprinted with customary crediting of the source. The American Phytopathological Society, 2000. germ plasm evaluation trials of intermediate wheatgrass at the Northern Great Plains Research Laboratory, Mandan, ND, individual plants originating from various populations were space planted on $0.9-\mathrm{m}$ centers. With tillage between plots, the individual plants can be maintained and evaluated for a number of agronomic characteristics and diseases. After 3 to 4 years of growth, disease affecting the persistence and long-term productivity of intermediate wheatgrass becomes obvious in some plots. The tillers of susceptible plants die in the center of the clonal plot, leaving a green ring of tillers around the edge of the plot that creates a "doughnut effect." Foot rotinfected intermediate wheatgrass tillers are found on the inside edge of this doughnut. Isolates of $F$. graminearum and $B$. sorokiniana have been obtained from the necrotic basal area of the culm of heading adult plants.

The use of host resistance is usually the most feasible and economical disease control strategy available for controlling many infectious diseases in forage crops (4). Recurrent selection techniques have been used to improve resistance to various diseases in cross-pollinated forage grasses $(5,7)$. The objective of this research was to select intermediate wheatgrass populations in the seedling stage of development for improved levels of resistance to foot rot diseases caused by $B$. sorokiniana and $F$. graminearum. Cvs. Reliant (1) and Manska (2) were used as base populations in this study because they have high levels of leaf spot resistance, forage yield, and nutritive quality relative to other current cultivars.
Considering that intermediate wheatgrass is a hexaploid $(2 n=6 x=42)$ and is cross pollinated, these cultivars, constituted from diverse parent clones, provide adequate genetic variation for selection.

\section{MATERIALS AND METHODS}

Isolates of $F$. graminearum and $B$. sorokiniana were obtained from the necrotic basal area of the culm of heading adult plants located in clonal plots at Mandan, ND. The identification of $F$. graminearum was confirmed at the Fusarium Research Center, University Park, PA (P. E. Nelson, personal communication). The same isolates of $F$. graminearum (91-11175-1) and B. sorokiniana (84-7105), stored in an ultra-low temperature freezer, were used throughout the study.

Sterile wheat (Triticum aestivum L.) seed overgrown with $F$. graminearum or $B$. sorokiniana was used to produce inoculum. The inoculum was prepared by placing 250 $\mathrm{g}$ of wheat seed with $100 \mathrm{ml}$ of distilled water in 1-liter flasks, autoclaving twice, and inoculating with a spore suspension of $F$. graminearum or B. sorokiniana obtained from cultures on $18 \%$ V8 juice agar $(18 \%$ V8 juice, $2 \%$ agar, and 2 g of calcium carbonate per liter). Flasks were incubated under cool white fluorescent light at $20^{\circ} \mathrm{C}$ for 2 weeks. The inoculated seed was kept loose by shaking the flasks by hand daily. All flasks of wheat inoculum for a particular fungus were combined and blended, resulting in a uniform inoculum.

In a preliminary study, it was determined with $F$. graminearum inoculations that the lowest seedling survival was obtained when inoculated wheat seed was mixed with the potting medium used to cover the intermediate wheatgrass seed. With $B$. sorokiniana inoculations, the lowest seedling survival was obtained when inoculated wheat seed was sprinkled over the seed before covering with potting medium. Thus, depending on the fungus, two methods of inoculum placement were used in order to obtain high selection pressure.

Seed of Reliant and Manska was planted and inoculated in trays $(21-\mathrm{cm}$ width by 31-cm length by $9-\mathrm{cm}$ depth) containing 6 $\mathrm{cm}$ of nonsterile potting medium composed of peat moss, vermiculite, and perlite $(1: 1: 1, \mathrm{vol} / \mathrm{vol} / \mathrm{vol})$. A template was used to mark seven $21-\mathrm{cm}$ rows spaced $3.8 \mathrm{~cm}$ apart on the potting medium of each tray. A 100-ml volume of inoculum was used to 
inoculate each tray. Depending on the fungus, the inoculum was placed either directly with the seed or mixed within the medium above the seed. Potting medium and $F$. graminearum inoculum were thoroughly mixed in a separate container to obtain a uniform mixture of inoculating medium. The same volume of medium was used to cover the seed in each tray and resulted in a planting depth of approximately $1 \mathrm{~cm}$. B. sorokiniana inoculum was uniformly sprinkled over the seed before covering the seed with potting medium. Trays were maintained in a glasshouse at $21 \pm 3^{\circ} \mathrm{C}$ during the light and $16 \pm 3^{\circ} \mathrm{C}$ during the dark. Sodium vapor lamps (400 W) were used to supplement natural sunlight and maintain a 14-h photoperiod. Four weeks after inoculation, the number of seedling plants was counted.

For each inoculation, an analysis of variance was conducted on the number of live plants and the arcsine-transformed percentage of live plants based on the uninoculated controls for each half-sib family (SAS version 6.12; SAS Institute, Cary, NC). The unselected base population (breeder seed of Reliant or Manska) included as a check in each inoculation was also included in each analysis. Dunnett's test $(P=0.05)$ was used to make paired comparisons between replicated half-sib family means and the base population in each inoculation. A randomized complete block design was used.

Inoculation of base population. Breeder seed of Reliant and Manska intermediate wheatgrass was used for the unselected base populations. Ten trays of Manska (250 seed/tray) and nine trays of Reliant (250 seed/tray) were inoculated with $F$. graminearum. Ten trays of Manska (250 seed/tray) and seven trays of Reliant (250 seed/tray) were inoculated with $B$. sorokiniana. Three uninoculated trays of each cultivar were included as a control to determine the number of seedlings obtained without inoculation pressure. Four weeks after inoculation, the number of seedlings was counted.

Approximately 300 seedling plants (range: 300 to 315) with no symptoms or mild symptom severity were selected for each cultivar for each fungus to establish four populations: Reliant/Fg (Reliant screened with $F$. graminearum), Reliant/Bs (Reliant screened with B. sorokiniana), Manska/Fg (Manska screened with $F$. graminearum), and Manska/Bs (Manska screened with $B$. sorokiniana). The four populations were space transplanted on $0.9-\mathrm{m}$ centers at isolated field locations so there would be natural intercrossing within a population but not among populations. Seed was harvested from each individual plant. Based on seed production and seed mass, 60 plant progenies (half-sib families) were selected from each population for further screening.

Inoculation of cycle-1 seed. Sixty halfsib families from each of the four cycle-1 populations were tested. An experimental unit consisted of 40 seeds from each halfsib family planted in row plots evenly spaced $(3.8 \mathrm{~cm}$ apart) in trays. Nine trays were required for each of three replicates. Trays were inoculated as described above. In addition to the inoculated trays, two uninoculated replicates for each half-sib family were included to determine the number of seedlings obtained without inoculation pressure. Original unselected breeder seed of Manska and Reliant, 21 rows (40 seeds per row) in three inoculated trays and 21 rows in three uninoculated trays, was also included in each inoculation to provide seedling plant survival data from the unselected base populations under the same conditions. Four populations with 60 half-sib families each were inoculated in four separate experiments. Half-sib families from base populations of Manska and Reliant originally selected for $F$. graminearum resistance (Manska/Fg and Reliant/Fg) were inoculated with $F$. graminearum. Half-sib families from base populations of Manska and Reliant originally selected for $B$. sorokiniana resistance (Manska/Bs and Reliant/Bs) were inoculated with $B$. sorokiniana.

Data from the two uninoculated replicates for each half-sib family were used to adjust the percentage of live seedlings that survived inoculation. The percentage of live seedlings was used to rank the 60 halfsib families from each population, based on the mean of three replicates, for resistance and to compare the half-sib families to the original base population. One hundred plants (five plants from each of the most resistant 20 half-sib families) from each of the four populations were selected and planted at four isolated field locations for intercrossing within populations. Seed yield and seed mass were recorded on individual plants to determine which half-sib families would be included in the next cycle of inoculations.

Inoculation of cycle-2 seed. Intercrossed individual plants selected from inoculated cycle- 1 half-sib families provided seed for the second cycling of halfsib families. Procedures were the same as those used for inoculating cycle-1 half-sib families. Cycle-2 seed was planted in rows (40 seeds per row): three replicates for inoculation and two replicates uninoculated. As with cycle 1, original unselected breeder seed was included to provide data on the unselected base populations under the same conditions. In all, 84 half-sib families from the Manska/Fg population and 94 half-sib families from the Reliant/Fg population were inoculated with $F$. graminearum, and 77 half-sib families

Table 1. Analyses of variance for half-sib families from four populations based on seedling plants surviving inoculation with Fusarium graminearum or Bipolaris sorokiniana

\begin{tabular}{|c|c|c|c|c|c|c|c|}
\hline \multirow[b]{2}{*}{ Population/treatment ${ }^{b}$} & \multirow[b]{2}{*}{ Source $^{c}$} & \multicolumn{2}{|c|}{ Cycle $1^{\mathrm{a}}$} & \multicolumn{2}{|c|}{ Cycle 2} & \multicolumn{2}{|c|}{ Cycle 3} \\
\hline & & df & Mean & df & Mean & df & Mean \\
\hline \multirow[t]{4}{*}{ Manska/Fg } & Replicate & 2 & $53^{*}$ & 2 & $108 * *$ & 2 & $61^{*}$ \\
\hline & Half-sib family & 60 & $32 * *$ & 84 & $27 * *$ & 99 & $30 * *$ \\
\hline & Error & 120 & 16 & 168 & 11 & 198 & 14 \\
\hline & Total & 182 & $\ldots$ & 254 & $\ldots$ & 299 & \\
\hline \multirow[t]{4}{*}{ Reliant/Fg } & Replicate & 2 & $8 \mathrm{NS}$ & 2 & $27 \mathrm{NS}$ & 2 & $164 * *$ \\
\hline & Half-sib family & 60 & $75^{* *}$ & 94 & $26^{* *}$ & 80 & $43^{* *}$ \\
\hline & Error & 120 & 15 & 188 & 15 & 160 & 16 \\
\hline & Total & 182 & $\ldots$ & 284 & $\ldots$ & 242 & $\ldots$ \\
\hline \multirow[t]{4}{*}{ Manska/Bs } & Replicate & 2 & $92 * *$ & 2 & $7 \mathrm{NS}$ & 2 & $120^{* * *}$ \\
\hline & Half-sib family & 60 & $47 * *$ & 77 & $31 * *$ & 86 & $40 * *$ \\
\hline & Error & 120 & 14 & 154 & 12 & 172 & 14 \\
\hline & Total & 182 & & 233 & & 260 & \\
\hline \multirow[t]{4}{*}{ Reliant/Bs } & Replicate & 2 & $77 * *$ & 2 & $994 * *$ & 2 & $300^{* * *}$ \\
\hline & Half-sib family & 60 & $37 * *$ & 81 & $48^{* *}$ & 102 & $41^{* *}$ \\
\hline & Error & 120 & 13 & 162 & 16 & 204 & 18 \\
\hline & Total & 182 & $\ldots$ & 245 & $\ldots$ & 308 & $\ldots$ \\
\hline
\end{tabular}

\footnotetext{
a Mean $=$ mean squares; NS $=$ non significant at $P=0.05 ; *=$ significant at $P=0.05 ; * *=$ significant at $P=0.01$.

${ }^{\mathrm{b}}$ Manska and Reliant = cultivar from which base population was selected. $\mathrm{Fg}=F$. graminearum and $\mathrm{Bs}=$ B. sorokiniana and indicate pathogen used for inoculation.

${ }^{c}$ Source of variation. Half-sib family $=$ base population included in the analysis with the half-sib families.
} 
from the Manska/Bs population and 81 half-sib families from the Reliant/Bs population were inoculated with $B$. sorokiniana. The adjusted percentage of live seedlings, based on the uninoculated checks for each half-sib family, was used to compare the half-sib families. For the next cycle of intercrossing, 110 plants (5 plants from each of the 22 most resistant half-sib families) from each of the four populations were randomized and planted in isolated field locations for intercrossing.

Inoculation of cycle-3 seed. Intercrossed individual plants selected from inoculated cycle- 2 half-sib families provided seed for the third cycle of half-sib families. Cycle-3 seed was planted in rows (40 seeds per row): three replicates for inoculation and two replicates uninoculated. Original unselected breeder seed was included. In all, 99 half-sib families from the Manska/Fg population and 80 half-sib families from the Reliant/Fg population were inoculated with $F$. graminearum, and 86 half-sib families from the Manska/Bs population and 102 half-sib families from the Reliant/Bs population were inoculated with $B$. sorokiniana. The adjusted percentage of live seedlings was used to compare the half-sib families. An initial report was published (8).

\section{RESULTS AND DISCUSSION}

Base population. Breeder seed of Reliant and Manska was screened for resistance to $F$. graminearum and $B$. sorokiniana. When adjusted to the number of seedlings surviving in the uninoculated treatment, $59 \%$ of the $F$. graminearuminoculated Reliant plants survived, and $17 \%$ (354) of the plants showed no symptoms. With Manska, 59\% of plants survived, and 14\% (326) of the plants showed no symptoms. When adjusted to the number of seedlings surviving in the uninoculated treatment, $63 \%$ of the $B$. sorokinianainoculated Reliant plants survived, and 55\% (889) of the plants showed no symptoms. With Manska, 53\% of the plants survived and $45 \%(1,042)$ of the plants showed no symptoms. Thus, the number of seedling plants surviving inoculation was similar for both pathogens but the number of symptomless plants was much higher for the B. sorokiniana inoculations compared with the $F$. graminearum inoculations. This indicates that the cultivars tested were apparently more resistant to $B$. sorokiniana than $F$. graminearum.

Cycle 1. Half-sib family, as a main effect in the analyses of variance, was significant in the four analyses conducted (Table 1), indicating significant differences for disease resistance among half-sib families for all four populations. When half-sib families were compared to the base population, only one half-sib family (Manska/ Bs population) was statistically more resistant (Table 2), indicating little improve- ment in disease resistance with one cycle of recurrent selection.

Cycle 2. Differences for disease resistance among half-sib families for all four populations was evident in all analyses (Table 1). When comparisons were made after the B. sorokiniana inoculations, six half-sib families from each population (Manska/Bs and Reliant/Bs) were more resistant than the base population, indicating that progress was made within those two populations for resistance to $B$. sorokiniana (Table 2). When half-sib families were compared to the base population for the $F$. graminearum inoculations, only one half-sib family was statistically more resistant (Manska/Fg population), indicating that slight progress was made for $F$. graminearum resistance.

Cycle 3. Differences for disease resistance among half-sib families for all four populations was evident in all analyses (Table 1). When half-sib families were compared to their base populations, progress was made in three populations. With the $F$. graminearum inoculations, 24 halfsib families were statistically more resistant than the base population, indicating progress in selecting disease resistance to $F$. graminearum was achieved with the Manska/Fg population (Table 2). Statistically, no progress was made with the Reliant/Fg population (Table 2). With the $B$. sorokiniana inoculations, 7 half-sib families were more resistant than the base population within the Reliant/Bs population, and 80 half-sib families were more resistant within the Manska/Bs population (Table 2). Thus, improved resistance to $B$. sorokiniana was more readily achieved than resistance to $F$. graminearum.

In order to visualize the results, the cycle-3 half-sib families for each population were divided in quartiles. When comparing the base population to the top three quartiles of the half-sib families in each popula-

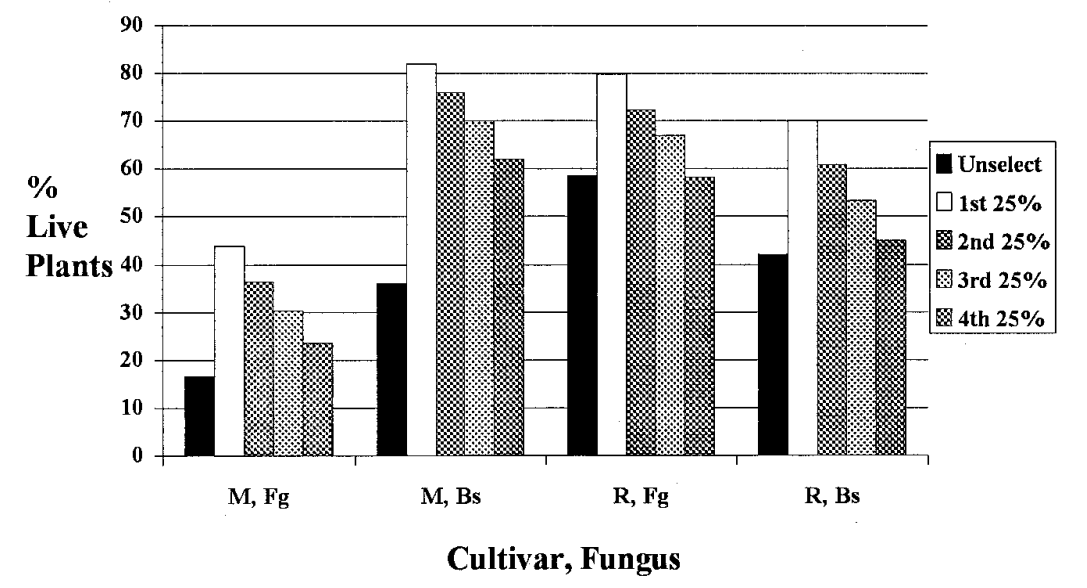

Fig. 1. Plants surviving inoculation with Fusarium graminearum and Bipolaris sorokiniana. Quartiles of cycle-3 half-sib family populations compared with the original base population included in the same inoculation. $\mathrm{M}=$ Manska intermediate wheatgrass, $\mathrm{R}=$ Reliant intermediate wheatgrass, $\mathrm{Fg}=$ F. graminearum, and $\mathrm{Bs}=B$. sorokiniana.

Table 2. Number of half-sib families for each population within each cycle that are significantly more resistant than the base population ${ }^{\mathrm{a}}$

\begin{tabular}{lcccc}
\hline & & & \multicolumn{2}{c}{ Resistant half-sib families $^{\mathbf{b}}$} \\
\cline { 4 - 5 } Population/treatment $^{\mathbf{c}}$ & Intercross & Total & Number & Percent \\
\hline Manska/Fg & Cycle 1 & 60 & 0 & 0 \\
Manska/Fg & Cycle 2 & 84 & 1 & 1 \\
Manska/Fg & Cycle 3 & 99 & 24 & 24 \\
Reliant/Fg & Cycle 1 & 60 & 0 & 0 \\
Reliant/Fg & Cycle 2 & 94 & 0 & 0 \\
Reliant/Fg & Cycle 3 & 80 & 0 & 0 \\
Manska/Bs & Cycle 1 & 60 & 1 & 2 \\
Manska/Bs & Cycle 2 & 77 & 6 & 8 \\
Manska/Bs & Cycle 3 & 86 & 80 & 0 \\
Reliant/Bs & Cycle 1 & 60 & 0 & 7 \\
Reliant/Bs & Cycle 2 & 81 & 6 & 7 \\
Reliant/Bs & Cycle 3 & 102 & 7 & 91 \\
\hline
\end{tabular}

a A Dunnett's test $(P=0.05)$ was used to make paired comparisons between the half-sib families and the unselected base population (breeder seed of Reliant or Manska intermediate wheatgrass), which was included in each inoculation as the check. Intercross $=$ cycle of intercrossing and Total $=$ total number of half-sib families.

${ }^{\mathrm{b}}$ Number and percent of half-sib families more resistant than the base population.

c Manska and Reliant = cultivar from which the population was originally selected. Fg $=$ Fusarium graminearum and $\mathrm{Bs}=$ Bipolaris sorokiniana and indicate pathogen used for inoculation. 


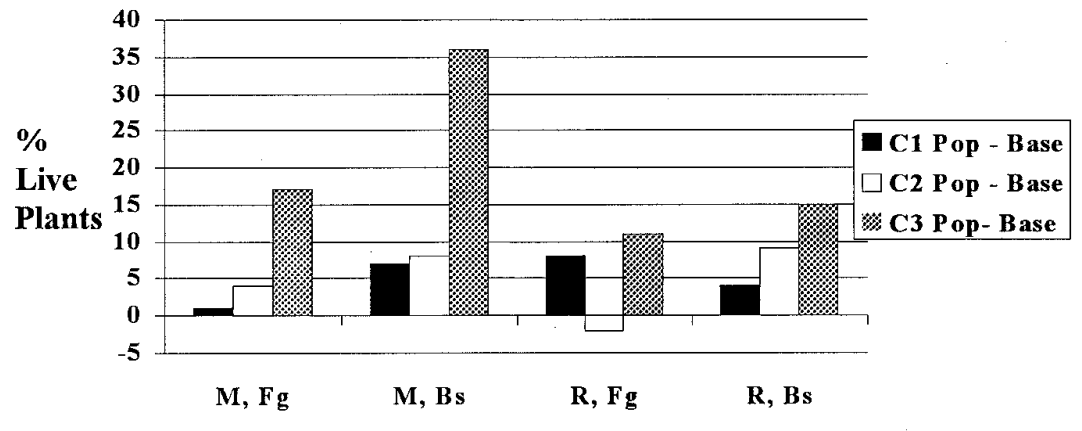

Cultivar, Fungus

Fig. 2. Progressive increase in the number of surviving seedling plants for each population demonstrated by the difference between the mean of the selected population and the mean of the base population included in the same inoculation. $\mathrm{M}=$ Manska intermediate wheatgrass, $\mathrm{R}=$ Reliant intermediate wheatgrass, $\mathrm{Fg}=$ Fusarium graminearum, and $\mathrm{Bs}=$ Bipolaris sorokiniana .

tion, all four populations had an increase in the percentage of live plants, indicating that progress for increased resistance was made (Fig. 1). Even when comparing the low ranking fourth quartile of the half-sib families to the base population, the resistance of the half-sib-families was at least equal to or better than the base population (Fig. 1).

Overall, there was a progressive increase in the percentage of seedling plants surviving inoculation, which would indicate an increase in resistance. Differences in the percentage of live plants between the overall mean of the half-sib families and the base population for each inoculation generally increased with each cycle, indicating that the percentage of resistant seedling plants was increasing in response to selection (Fig. 2). The greater increase in resistance from cycle 2 to cycle 3 than from cycle 1 to cycle 2 , in most instances, might be explained by quantitative inheritance of resistance and the number of resistance genes required before resistance is expressed.

The Manska/Bs and Reliant/Bs populations had consistent improvement in seedling survival in comparison to the base population. The percentage of half-sib families that were significantly more resistant than the base population increased from cycle 1 to cycle 2 and from cycle 2 to cycle 3 for both populations. In cycle 3,7 to $91 \%$ of the half-sib families were more resistant than the base populations (Table 2 ). The difference between the mean of the
Manska/Bs population and the base population increased from 7 to 8 to 36 for cycle 1 , cycle 2 , and cycle 3 , respectively (Fig. 2). Manska, a cultivar with a broad genetic base (2), appears to have the potential for higher levels of $B$. sorokiniana resistance than Reliant. This is based on the Manska/Bs population producing a greater percentage of half-sib families significantly more resistant than the base population (Table 2). The difference between the mean of the Reliant/Bs population and the base population increased from 4 to 9 to 15 for cycle 1 , cycle 2 , and cycle 3 , respectively (Fig. 2). This would indicate that selection for increased resistance was effective.

Although improvement in resistance was realized with the $F$. graminearum inoculations, the results were not as consistent as with the Manska/Bs and Reliant/Bs populations. The number of half-sib families that were significantly more resistant than the base population increased from cycle 1 to cycle 2 and from cycle 2 to cycle 3 for the Manska/Fg population, but not for the Reliant /Fg population. In cycle 3, 24\% of the half-sib families were more resistant than the base populations for the Manska/Fg population (Table 2). Similar to results found in the Manska/Bs population, Manska appears to have the potential for higher levels of $F$. graminearum resistance than Reliant, because half-sib families significantly more resistant than the base population were obtained with the Man$\mathrm{ska} / \mathrm{Fg}$ population. Thus, the level of re- sistance to $F$. graminearum in the base population is apparently lower than resistance for B. sorokiniana or is more difficult to select.

Overall, recurrent phenotypic selection among and within half-sib families of intermediate wheatgrass for seedling resistance to both pathogens was effective for three populations. Increased resistance to $B$. sorokiniana and $F$. graminearum in these intermediate wheatgrass populations is important for plant establishment in the field.

\section{ACKNOWLEDGMENTS}

We thank D. Wetch and B. Wald for technical assistance.

\section{LITERATURE CITED}

1. Berdahl, J. D., Barker, R. E., Karn, J. F., Krupinsky, J. M., Haas, R. J., Tober, D. A., and Ray, I. M. 1992. Registration of 'Reliant' intermediate wheatgrass. Crop Sci. 32:1072.

2. Berdahl, J. D., Barker, R. E., Karn, J. F., Krupinsky, J. M., Ray, I. M., Vogel, K. P., Moore, K. J., Klopfenstein, T. J., Anderson, B. E., and Haas, R. J. 1993. Registration of 'Manska' pubescent intermediate wheatgrass. Crop Sci. 33:881.

3. Braverman, S. W. 1986. Disease resistance in cool-season forage range and turf grasses II Bot. Rev. 52:1-112.

4. Casler, M. D., and Pederson, G. A. 1996. Host resistance/tolerance and its deployment Pages 475-507 in: Pasture and Forage Crop Pathology. S. Chakroborty, K. T. Leath, R. A. Skipp, G. A. Pederson, R. A. Bray, G. C. M Latch, and F. W. Nutter, Jr., eds. Am. Soc. Agron. Madison, WI.

5. Engqvist, G. 1997. Breeding for resistance in grasses and forage legumes. Sver. Utsadesforen. Tidskr. 107:100-110.

6. Farr, D. F., Bills, G. F., Chamuris, G. P., and Rossman, A. Y. 1989. Fungi on Plants and Plant Products in the United States. APS Press, St. Paul, MN.

7. Jonsson, H. A. 1997. Plant breeding of herbage grasses. Sver. Utsadesforen. Tidskr. 107:64-76.

8. Krupinsky, J. M., and Berdahl, J. D. 1996. Screening method for root rot resistance in intermediate wheatgrass. (Abstr.) Phytopathology 86:(11, Suppl.) S41.

9. Lawrence, T., and Warder, F. G. 1979 Evaluation of twenty grass populations as irrigated hay crops for southwestern Saskatchewan. Can. J. Plant Sci. 59:691-700.

10. Leath, K. T., Welty, R. E., Pratt, R. G., and Sonoda, R. M. 1996. Pasture/forage crops and diseases in the United States. Pages 33-59 in: Pasture and Forage Crop Pathology. S. Chakroborty, K. T. Leath, R. A. Skipp, G. A Pederson, R. A. Bray, G. C. M. Latch, and F. W. Nutter, Jr., eds. Am. Soc. Agron. Madison, WI.

11. Sprague, R. 1950. Diseases of Cereals and Grasses in North America. Ronald Press Co., New York. 